\title{
Near-infrared spectroscopy in NGC 7538 ${ }^{\star}$
}

\author{
E. Puga ${ }^{1}$, A. Marín-Franch ${ }^{2}$, F. Najarro ${ }^{1}$, A. Lenorzer ${ }^{2}$, A. Herrero ${ }^{2}$, J. A. Acosta Pulido ${ }^{2}$, L. A. Chavarría ${ }^{3}$, A. Bik ${ }^{4}$, \\ D. Figer ${ }^{5}$, and S. Ramírez Alegría ${ }^{2}$
}

${ }^{1}$ Centro de Astrobiología (CSIC-INTA), Ctra. de Torrejón a Ajalvir km-4, 28850, Torrejón de Ardoz, Madrid, Spain e-mail: elena@damir.iem.csic.es

2 Instituto de Astrofísica de Canarias, C/ vía Láctea s/n, 38200, La Laguna, Spain

Harvard-Smithsonian Center for Astrophysics, 60 Garden Street, Cambridge, MA 02138, USA

4 Max-Planck-Institut für Astronomie, Königstuhl 17, 69117, Heidelberg, Germany

5 Rochester Institute of Technology, 54 Lomb Memorial Drive, Rochester NY 14623, USA

Received 14 September 2009 / Accepted 18 February 2010

\section{ABSTRACT}

\begin{abstract}
Aims. The characterisation of the stellar population in young high-mass star-forming regions allows fundamental cluster properties like distance and age to be constrained. These are essential when using high-mass clusters as probes for conducting Galactic studies. Methods. NGC 7538 is a star-forming region with an embedded stellar population unearthed only in the near-infrared (NIR). We present the first near-infrared spectro-photometric study of the candidate high-mass stellar content in NGC 7538 . We obtained $H$ and $K$ spectra of 21 sources with both the multi-object and long-slit modes of LIRIS at the WHT, and complement these data with subarcsecond $J H K_{\mathrm{s}}$ photometry of the region using the imaging mode of the same instrument.

Results. We find a wide variety of objects within the studied stellar population of NGC 7538. Our results discriminate between a stellar population associated to the H II region, but not contained within its extent, and several pockets of more recent star formation. We report the detection of $\mathrm{CO}$ bandhead emission toward several sources, as well as other features indicative of a young stellar nature. We infer a spectro-photometric distance of $2.7 \pm 0.5 \mathrm{kpc}$, an age spread in the range $0.5-2.2 \mathrm{Myr}$ and a total mass $\sim 1.7 \times 10^{3} M_{\odot}$ for the older population.
\end{abstract}

Key words. stars: early-type - stars: formation - stars: pre-main sequence - infrared: stars

\section{Introduction}

Clusters of total mass $>10^{3} M_{\odot}$ are the natural habitat of highmass stars. Whether motivated by the study of the formation, evolution of feedback effects of high-mass stars themselves (Bik et al. 2010; Martins et al. 2009; Puga et al. 2009), or as probes to investigate the physics and chemistry evolution of the Galaxy (Messineo et al. 2009; Davies et al. 2009), NIR spectroscopic studies have been proven powerful at unveiling and characterising the complete high-mass stellar content within massive clusters. Although new NIR surveys, such as UKIDSS and VISTA, will surely uncover new obscured massive clusters, some clusters that are already known from optical observations harbour hidden high-mass stars only accessible at NIR wavelengths (e.g. Cyg OB2, Knödlseder 2000).

NGC 7538 (aka Sh 2-158) is a visible H II region in the Perseus spiral arm and a site of active star formation. An early detection of several luminous NIR and far-IR sources in the vicinity of this region (Campbell \& Persson 1988) hinted at a rather massive nature. In particular, NGC 7538 IRS 1 is a highmass $\left(\sim 30 M_{\odot}\right)$ protostar with a CO outflow, known to power the ultra-compact (UC)H II region NGC $7538 \mathrm{~A}$. It also has an associated linear methanol maser structure, which might trace a Keplerian-rotating circumstellar disk (Pestalozzi et al. 2004). Recently, $6.7 \mathrm{GHz}$ methanol masers have been detected toward

^ Based on observations made with the WHT operated on the island of La Palma by the Isaac Newton Group in the Spanish Observatorio del Roque de los Muchachos of the Instituto de Astrofísica de Canarias. the nearby objects NGC 7538 IRS 9 and NGC 7538 S, tracing other young and embedded massive protostars (Pestalozzi et al. 2006).

Although this region has been widely inspected at long wavelengths (mostly in the submillimetre window) and optical spectroscopy for two stars has been obtained (Russeil et al. 2007), only a few detailed near-IR photometric studies of the stellar population in NGC 7538 have been conducted (Balog et al. 2004; Ojha et al. 2004; McCaughrean et al. 1991). At subarcsecond resolution, the region breaks down into several areas of interest (see Fig. 1): (i) the presumed powering cluster, centred on the location of IRS 6; (ii) the subcluster sitting amidst the bright NIR reflection nebula (IRS 5); (iii) the cluster located at the southern rim of the optical H II region (IRS 4); (iv) the active region around IRS 1-3; (v) an embedded stellar cluster at the location of NGC $7538 \mathrm{~S}$, only detected at wavelengths longward of $3.6 \mu \mathrm{m}$; and (vi) the IRS 9 reflection nebula. Surprisingly, the stellar density detected in the NIR is highest at the southern rim of the dust bubble, which bounds the optical H II region (Balog et al. 2004) between regions (iii) and (iv). The distance to NGC 7538 has been estimated several times and values between 2.2 and $3.5 \mathrm{kpc}$ have been reported (Moreno \& Chavarria-K. 1986; Israel et al. 1973).

In this article, we present NIR spectra of a sample of candidate high-mass stars within NGG 7538 together with subarcsecond NIR photometry. We describe the observations and data reduction in Sect. 2. In Sect. 3 we analyse the spectro-photometric information of a sample of candidate high-mass stars. Section 4 


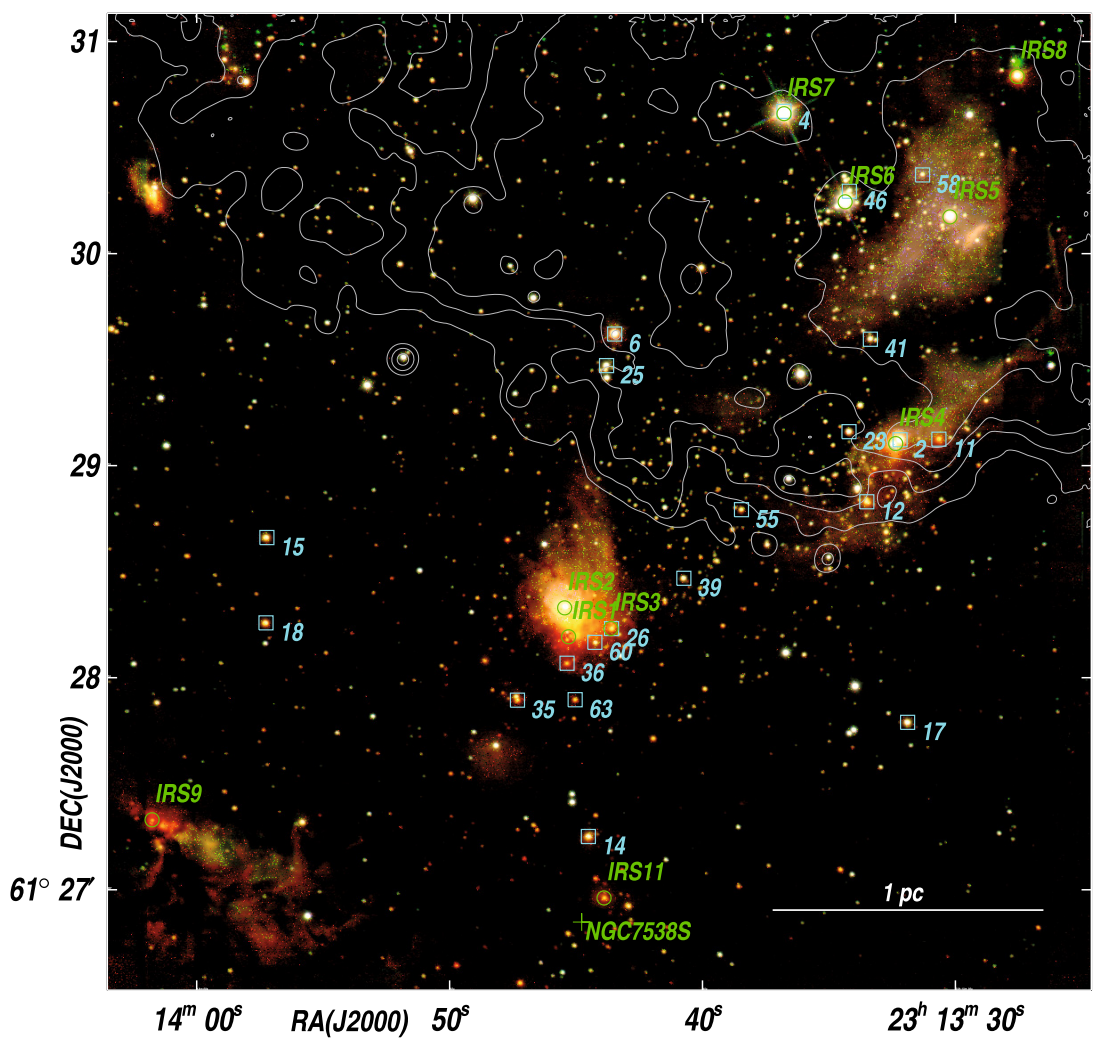

Fig. 1. Colour composite image of NGC 7538 obtained with LIRIS (blue: $J$, green: $H$, red: $K_{\mathrm{s}}$ ). Overlayed contours depict the extension of the $\mathrm{H}$ II region in the DSS/POSSII-F Red map. Stars observed with the LIRIS-MOS mode are labelled in cyan. Object \#4 was observed with the same instrument in long-slit mode. The green circles and cross are 2MASS point sources of interest.

is dedicated to estimating the distance, age, and mass of the cluster powering the H II region. Finally, we conclude in Sect. 5 with a brief summary.

\section{Observations and data reduction}

\subsection{NIR photometry}

We conducted $J H K_{\mathrm{s}}$ imaging observations of the field around NGC 7538 with LIRIS (Acosta Pulido et al. 2003; Manchado et al. 2004), mounted at the Cassegrain focus of the William Herschel Telescope in La Palma. Observations were obtained on 2006 July 21 as part of the MASGOMAS programme that comprised a total of 45 young clusters in the Milky Way (see Marín-Franch et al. 2009, for a description of the programme). The average seeing during the imaging observations of this re-

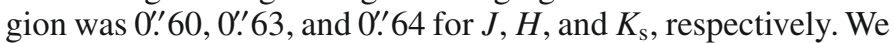
followed the procedure described in Marín-Franch et al. (2009) for the reduction and PSF extraction of the point-like sources in the field. The saturation limit for the LIRIS photometry corresponds to $K_{\mathrm{s}} \sim 10.5 \mathrm{mag}$. For brighter objects, we complemented the photometry with the 2MASS point-source catalogue values that had their photometric errors determined.

\subsection{NIR spectroscopy}

Follow-up spectroscopic observations of NGC 7538 were obtained in 2007 September 20 and 2008 August 19 using the multi-object spectroscopic (MOS) mode of the LIRIS Instrument. The average seeing during both nights was $0 \prime^{\prime} 9$. The designed mask comprised 20 slitlets of 0.8 in width and $6^{\prime \prime}$ in length. We used the low-resolution grism $(\lambda / \Delta \lambda \sim$ 700) that covers the $H$ and $K$ band simultaneously and the medium-resolution grisms in the $H$ and $K$ bands with $\lambda / \Delta \lambda \sim$ 2500 and $\lambda / \Delta \lambda \sim 1700$, respectively. The slitlet mask was oriented along a $\mathrm{PA}=142^{\circ}$ and centred at $\alpha_{2000}=23^{\mathrm{h}} 13^{\mathrm{m}} 39^{\mathrm{s}}$, $\delta_{2000}=+61^{\circ} 28^{\prime} 60^{\prime \prime}$.

We obtained the observations following a classic ABBA telescope nodding pattern with an offset of 2 .'5. In the case of two slitlets, the spectra appeared contaminated by other sources that fell close to one of the nodding positions (position B). Therefore, we extracted those specific spectra on only half of the stacked frames (at the position A). Observations of the AOV standard star HD223386 were obtained in the different configurations in order to remove any telluric features due to the Earth's atmosphere.

The data were reduced using LIRISDR $^{1}$, a specific data reduction package developed under the IRAF environment by the LIRIS team. The routines available for the LIRIS MOS mode make use of the a-priori mask design information to trace the slitlets and their limits in the spatial direction in a uniformly illuminated frame. Special care was taken to make the flat-field correction. The limited spatial extent of each slitlet may include detector defects at comparable or larger spatial scales, which are not detected within individual flat-field slitlets. We circumvented the problem by coadding the spectral response information of all apertures into a unique monodimensional response, after shifting by an appropriate offset along the spectral axis. This response was modelled using a high order spline function. The pixel-to-pixel correction was determined for each slitlet from the extracted flat-field spectrum divided by the modelled response.

We performed a standard subtraction of consecutive $A B$ pairs to remove the sky background. Individual flatfielding,

\footnotetext{
${ }^{1}$ http://www.iac.es/project/LIRIS
} 

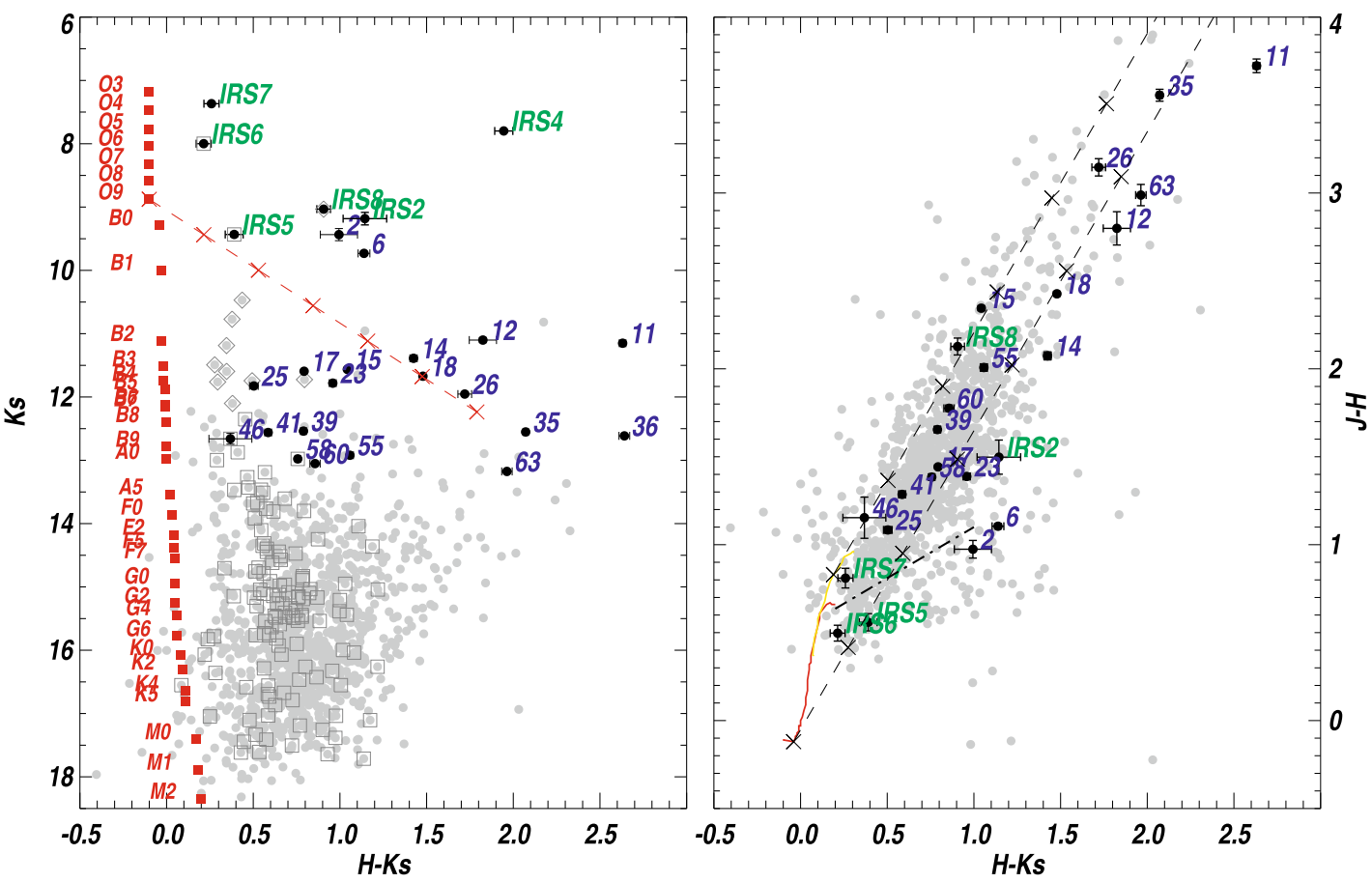

Fig. 2. (Left panel) CMD of the stellar content within the field of view of the left panel. The red squares represent the main sequence (MS) of dwarf stars without extinction at a heliocentric distance of $2.7 \mathrm{kpc}$. The red discontinuous line represents the reddening vector for an $\mathrm{O} 9 \mathrm{~V}$ star, with intervals of $A_{V}=5$ mag indicated by crosses. Dark squares represent objects located at a projected distance less than 30" from IRS 6. (Right panel) Colour-colour diagram of NGC 7538. Source \#36 lies at [4.84, 2.64], out of the scale of the figure. The dashed lines indicate the direction of extinction according to Rieke \& Lebofsky (1985) with overlayed intervals of $A_{V}=5$ mag indicated by crosses. The dash-dotted line represents the T Tauri locus of Meyer et al. (1997).

retracing, resizing, and extraction for each star in every subtracted frame followed. Argon and Xenon arc frames were used to determine the wavelength calibration ( $\mathrm{rms}<1 \AA$ ). The resulting spectra were coadded weighting by the relative number of counts to provide a final spectrum of each star.

The wavelength calibrated spectra were divided by a normalised reference spectrum to remove the telluric absorption features. This normalised reference spectrum was generated from the observed spectra of the A0V standard star, divided by a Vega model spectrum convolved to the correspondent spectral resolution. For this, we constructed an IDL routine that iteratively fits the components due to the instrument response, an atmosphere's transmissivity template, and the Vega model spectrum.

Source \#4 was observed with the LIRIS long-slit mode in the ranges $1.92-2.4 \mu \mathrm{m}$ and $1.52-1.78 \mu \mathrm{m}$ in the nights of 2007 September 21 and 2008 June 27, respectively. The intermediateresolution grisms provided spectral resolutions of $\lambda / \Delta \lambda \sim 1700$ and $\lambda / \Delta \lambda \sim 2500$. In this case, we traced the spectrum using the continuum of the source and applied an optimal weight to extract the flux along the spatial direction. The standard star HD223386 was observed in the same configurations to obtain the telluric correction.

\section{Results}

\subsection{Colour-magnitude and colour-colour diagrams}

The left and right panels of Fig. 2 show the colour-magnitude diagram (CMD) and the colour-colour diagram (CCD) of the field around NGC 7538. In the figure, we have only depicted those objects that have LIRIS counterparts in the $H$ and $K_{\mathrm{s}}$ bands and in the three channels for the CMD and CCD, respectively.
The 2MASS source IRS 4 appears to be resolved into two components in our LIRIS images (see also Ojha et al. 2004). The brighter source to the southeast is saturated in our $K_{\mathrm{s}}$ map, while the northwest source \#2 is one of the brightest in the field. Therefore, we used our LIRIS photometry for source \#2, while still displaying the 2MASS integrated photometry of these two point-like sources in our CMD (IRS 4). IRS 4 is not detected in the 2MASS $J$ band survey, therefore, it is important to notice that it shows $J-H \geq 1.34$ and $H-K_{\mathrm{s}}=1.94 \pm 0.05$, pointing to a strong NIR excess.

This paper aims at identifying the high-mass stellar content of a Galactic massive cluster, thus we selected the candidates for spectroscopic follow-up using a NIR photometric criterion. We looked for reddened bright objects in high stellar density regions. The production design specifications of the slitlet mask hinder a complete study of extremely crowded regions. A geometrical limitation is also imposed by the LIRIS multi-object mode. The spectral coverage for each object depends on the position of the corresponding slit relative to the centre of the chip in the dispersion direction. Therefore, the follow-up sources must be selected in a preferred orientation along the sky. Our CCD brings out the strong variations in extinction toward the sources selected for follow-up spectroscopy.

\subsection{Spectral classification}

We present the higher resolution spectra of the selected highmass star candidates in Figs. 3 and 4. The lower resolution spectra are presented in Fig. 5. The inspection of the NIR spectra of the 21 objects observed in NGC 7538 suggests a classification into the six major groups exemplified in Fig. 6. 

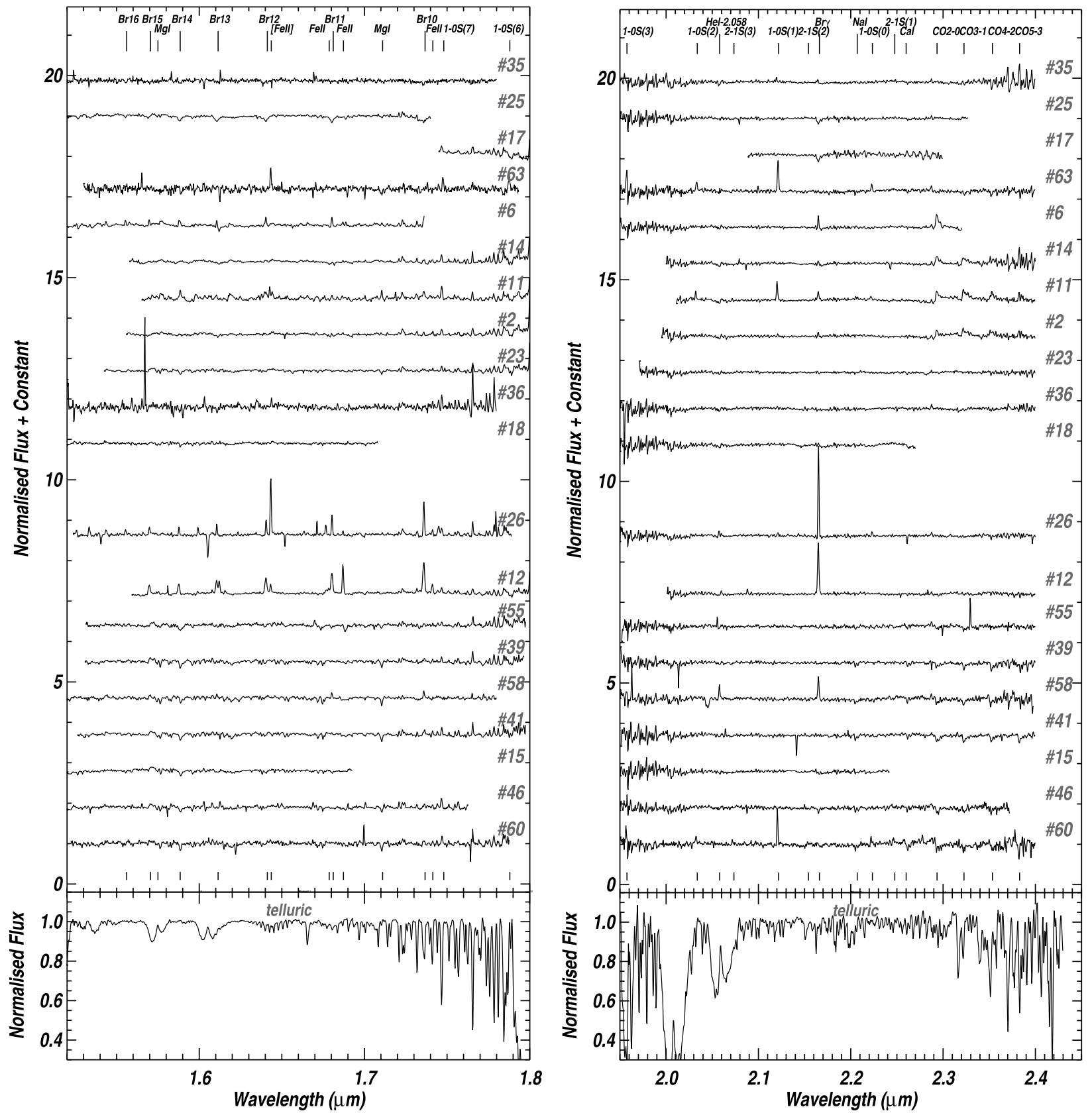

Fig. 3. (Left panel) $H$-band normalised spectra of the 20 sources with $\operatorname{MOS}(R \sim 2500)$. A normalised template telluric spectrum is shown in the bottom panel. (Right panel) $K$-band normalised spectra of the 20 sources with MOS $(R \sim 1700)$. A normalised template telluric spectrum is shown in the bottom panel.

Early-type stars: only 3 objects within our candidate sample exhibit atmospheric spectral features in absorption, but none of them show traces of helium. This proves that sources \#35, \#25, and \#17 are B-type stars. Considering the measured Br-11 and Br $\gamma$ EWs, we classified the early-type stars following the schemes described in Hanson et al. (1996) and Hanson et al. (1998). The obtained spectral types range between B1.5 and B9V.

IRS 6 and IRS 5 are very bright sources in the field of NGC 7538 that we did not include in our NIR spectroscopic follow-up. These objects were already classified by Russeil et al. (2007) according to the spectral features detected in their blue spectra. However, the strength of the HeI and HeII lines on these published spectra indicate that, opposite to what the authors state in the article, IRS 6 is of earlier spectral type than IRS 5.
To investigate this, we performed spectroscopic observations in September 2009 with ISIS at the William Herschel Telescope, confirming that the spectral classification of Russeil et al. (2007) is swapped for these two particular objects. This revised classification solves the disparity in the estimates of the cluster distance derived from each star, as discussed later in Sect. 4.1.

$\mathrm{H}_{2}$ emission sources: NIR ro-vibrational emission from $\mathrm{H}_{2}$ is detected toward source \#63. This source is located in the vicinity of the active IRS 1-3 region and amidst the strong extended $\mathrm{H}_{2}$ emission reported by Davis et al. (1998) and Bloomer et al. (1998) and attributed to IRS 1/2 (Kraus et al. 2006). The molecular hydrogen emission indeed has a thermal origin, pointing to the presence of shocks produced by outflows. The spectra of 

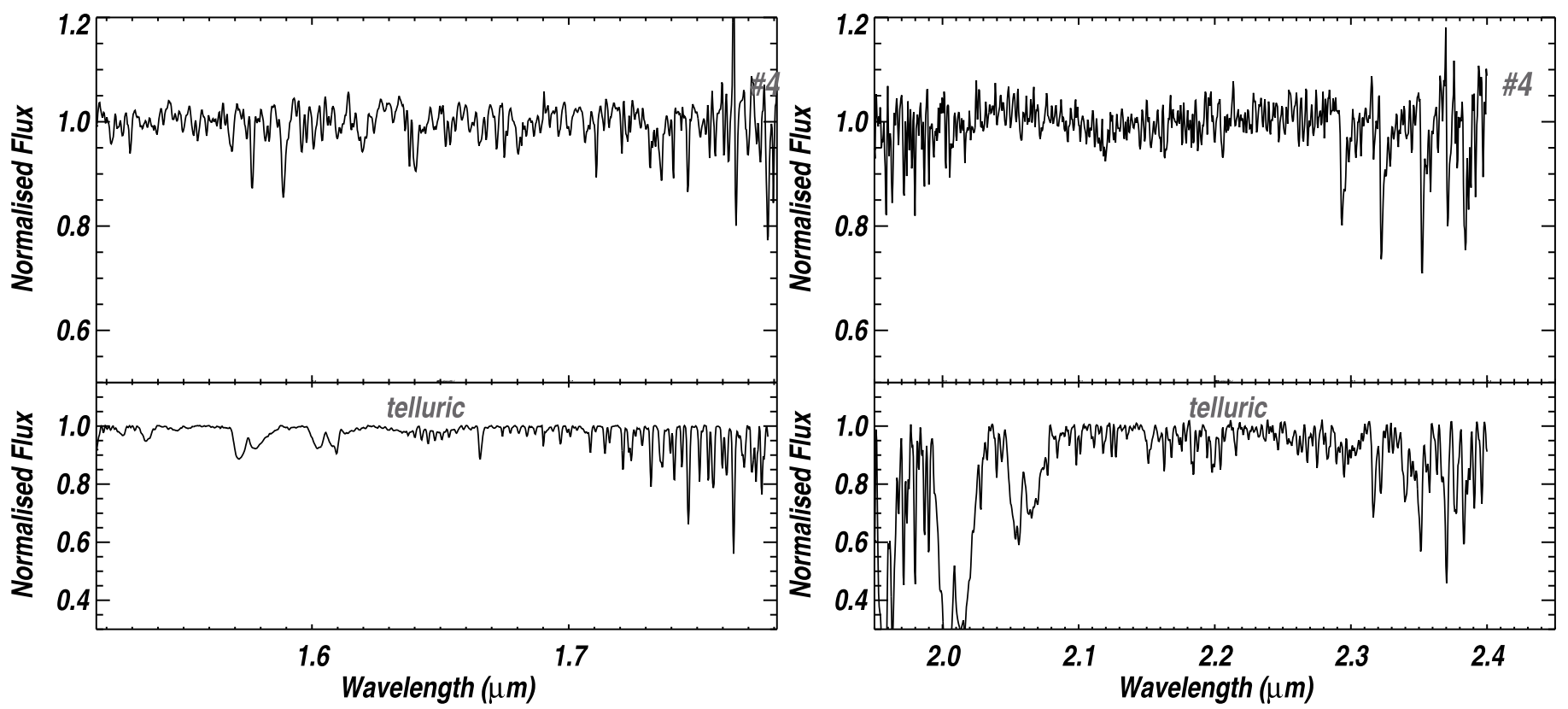

Fig. 4. (Left panel) $H$-band normalised spectrum of source \#4 obtained with long-slit $(R \sim 2500)$. (Right panel) $K$-band normalized spectrum of the same source with long-slit $(R \sim 1700)$. A normalised template telluric spectrum is shown below.

sources \#60, \#11, and \#2 also show traces of $\mathrm{H}_{2} 1-0 \mathrm{~S}(1)$ emission. However, this spectral feature has a nebular origin for the earlier case or it is not a dominant feature for the other two.

CO bandhead emission sources: objects \#6 and \#14 show $\mathrm{CO}$ first-overtone emission in their K-band spectra. These $\mathrm{CO}$ bandheads are emitted by neutral material with temperatures in the range $2000-5000 \mathrm{~K}$ and densities of $\sim 10^{10} \mathrm{~cm}^{-3}$ (Scoville et al. 1979). The CO bandhead and $\mathrm{Br} \gamma$ emissions are spectral features commonly displayed by YSOs (Bik et al. 2006). Surprisingly, their $H$-band spectra show both nebular emission and Brackett features in absorption. These two candidate YSOs are fairly isolated: \#6 lies in the centroid of a trunk-like structure at the rim of the Sh 2-158 H II region in the IRAC maps, whereas \#14 belongs to the embedded cluster detected at the location of NGC $7538 \mathrm{~S}$ or region (v). Sources \#11 and \#2, located respectively $13^{\prime \prime}$ and $2^{\prime \prime}$ away from IRS 4, show Br $\gamma$ and CO first-overtone emission in their spectra. Contamination by IRS 4 must be considered, particularly in the spectrum of source \#2.

Featureless sources: sources \#23, \#36, and \#18 are identified as featureless. The NIR spectra of these objects could resemble those of low-mass Class I objects in Greene \& Lada (1996) or even the Herbig Ae stars found by Rodgers (2001).

Brackett+Fe emission sources: two objects (\#26 and \#12) in the field show a peculiar spectrum with the Brackett series in emission and several FeII lines. Source \#12 shows broad Br1510 features (average $F W H M$ of $220 \mathrm{~km} \mathrm{~s}^{-1}$ ) and FeII lines at 1.688, 1.742, 2.061, and $2.089 \mu \mathrm{m}$ and [FeII] line emission at $1.644 \mu \mathrm{m}$. For classical Be stars, Fe II emission in the $K$ band, while $\mathrm{MgII}$ is not detected, is observed in stars of spectral type B4-B7 (Clark \& Steele 2000). A hint of CO bandhead emission is observed in its $K$-band spectrum. Due to the forbidden line emission in its spectrum, we classify \#12 as a Herbig Be.

The $\mathrm{CO}$ bandhead and broad $\mathrm{Br} \gamma$ emission are distinctive features associated with YSOs (Bik et al. 2006). The spectrum of object \#26 (aka IRS 3) shows Br17-10, FeII lines, and prominent [FeII] emission at $1.553,1.644$, and $1.677 \mu \mathrm{m}$. The ratio of [FeII] 1.553 to $1.644 \mu \mathrm{m}$ flux for this source $(\sim 0.33)$ is indicative of a high-density environment $n_{\mathrm{e}}>10^{4} \mathrm{~cm}^{-3}$ (Hamann et al. 1994). This is not surprising, since \#26 appears reddened in our CCD by an $A_{\mathrm{V}} \sim 27$ mag. Source \#26 therefore appears deeply buried and located at only $0.2 \mathrm{pc}$ in projected distance from the high-mass protostars IRS 1/2 (Bloomer et al. 1998). The presence of [FeII] lines toward \#26, together with the detection of $\mathrm{H}_{2}$ emission in the same spectra, suggest there are dissociative J-type shocks, tracing flow activity toward \#26 and confirming the youth of this source. Source \#26 has an associated H II region studied in radio recombination lines and detected continuum counterparts at 3.6 and $2 \mathrm{~cm}$ (Sewilo et al. 2004). These author's inferred Lyman continuum photon rate corresponds to that of an early B-type dwarf. This result is in good agreement with our photometry and the dereddened $F(\mathrm{HeI} 2.058) / F(\mathrm{Br} \gamma)=0.073$, which indicates temperatures lower than those produced by O-type stars (Benjamin et al. 1999). We classify \#26 as an H II region powered by a B star with ongoing flow activity. The presence of FeII lines may indicate that, although much of the emission is from an $\mathrm{H}$ II region, the object is still rather young and surrounded by circumstellar material.

There is a strong similarity between the $H$ - and $K$-band spectra of \#26 and \#12 with some LBVs at low excitation (e.g. Geballe et al. 2000), showing Brackett lines and permitted and forbidden FeII lines in emission. However, the low luminosity $\left(\leq 10^{4} L_{\odot}\right)$ of these objects, together with the absence of MgII and SilI lines, goes against any LBV classification. This should be regarded as a warning against misidentifications in LBV infrared spectroscopic searches in our Galaxy.

CO bandhead absorption sources: seven objects within the sample show typical spectral features of cool stars. Our classification of these sources follows the prescriptions of Winston et al. (2009) and Bik et al. (2010) for late-type stars. This comparative method uses primarily the $\mathrm{MgI}$, NaI, and CaI atomic lines as diagnostics for the temperature and the $\mathrm{CO}$ first overtone 

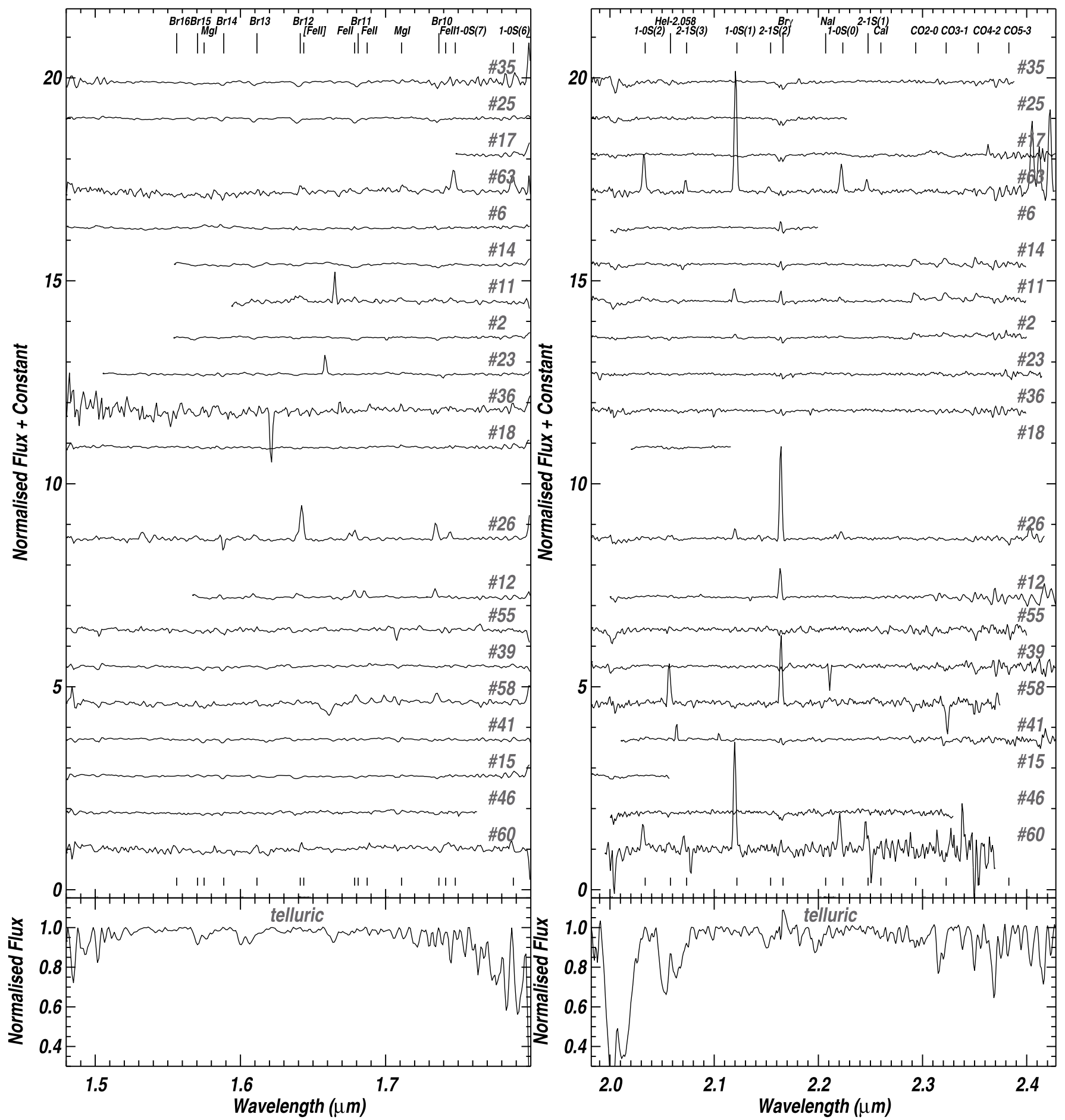

Fig. 5. $H$ - and $K$-band normalised spectra of the 20 sources with MOS $(R \sim 700)$. A normalised template telluric spectrum is shown in the bottom panel.

absorption bands as luminosity indicator. This classification carries an uncertainty of about \pm 1 spectral subclass. Stars \#46 and \#4 show spectral features compatible with a K0III, whereas star \#60 is classified as a K2III. Sources \#55, \#39, \#58, and \#41 have shallower $\mathrm{CO}$ absorption bands than giants and yet are deeper than dwarfs, resembling that of luminosity class IV. Therefore, these objects are considered pre-main sequence stars (PMS).

Despite the small offsets between the nodding positions and the narrow aperture used for extracting the spectra, nebular H II emission appears strongly in the spectra of sources \#58 and \#41. These objects are located in the line of sight of areas where a strong gradient in the nebular emission is detected in our NIR maps. Therefore, these lines should be considered with caution.

A summary of the photometric and spectroscopic characterisation of NGC 7538's stellar content is compiled in Table 1.

The spectral type classification allows the estimate of the extinction toward several sources in NGC 7538. The visual extinctions that appear in Col. 8 of Table 1 were calculated from the spectral type classification and corresponding stellar colours, considering the Rieke \& Lebofsky (1985) extinction law. The intrinsic colours of O-type dwarfs were taken from Martins \& Plez (2006), while those for B dwarfs and giants were extracted 

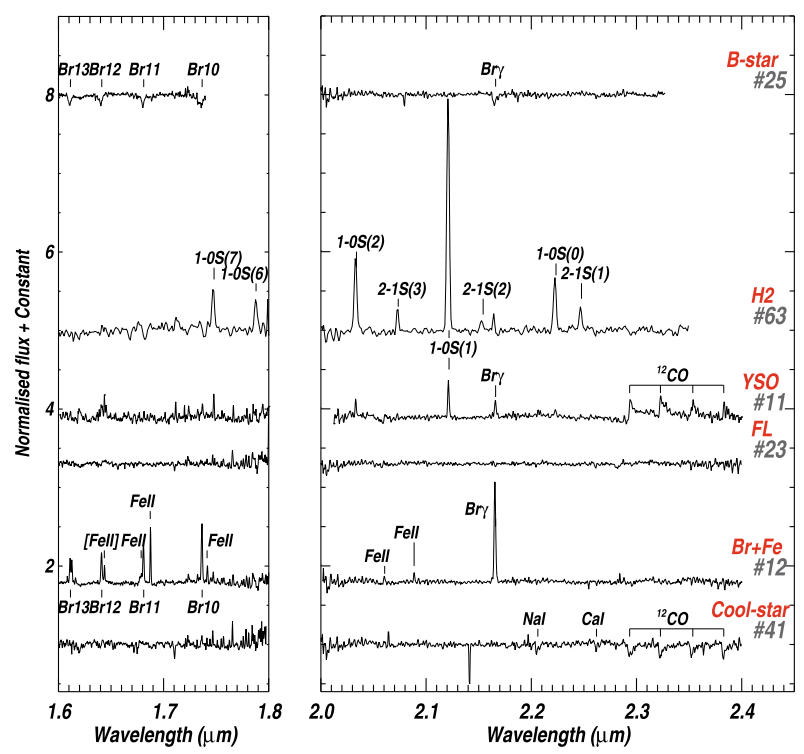

Fig. 6. Sample of representative spectra in NGC 7538. B-star: B-type star; $\mathrm{H} 2$ : dominant $\mathrm{H}_{2}$ lines; YSO: young stellar object; FL: featureless spectrum; $\mathrm{Br}+\mathrm{Fe}$ : Brackett and FeII emission lines; Cool-St: cool star.

from Tokunaga (2000). For PMS stars, we considered the intrinsic colours reported by Kenyon \& Hartmann (1995). Absolute magnitudes are taken from Martins \& Plez (2006) for O stars.

\subsection{Infrared excess}

We have complemented our NIR photometry with IRAC/Spitzer mid-infrared (MIR) data for this region (Chavarria et al. private communication) in order to study the infrared excess toward our follow-up sources. Figure 7 shows the spectral energy distribution (SED) of the spectroscopically identified young sources within NGC 7538.

We fitted the LIRIS and IRAC flux distributions with (i) a Kurucz model depicted with a solid line for objects with a spectral type classification of according temperature and surface gravity; (ii) a single black-body distribution represented by a broken line for those sources whose spectral type could not be constrained. Only in the first case were the fluxes dereddened following the Fitzpatrick (1999) parametrisation. The total luminosity for each object was computed by integrating the area below the respective model fit, and it is considered a lower limit for objects that display any excess.

Object \#63 shows both NIR and MIR excess, although the $8.0 \mu \mathrm{m}$ IRAC map appears saturated at this location. The large IRAC colour [3.6] $-[4.5]=0.9 \mathrm{mag}$ hints at a Class I source. Martins et al. (2009) show that the $\mathrm{H}_{2}$-dominated sources in the RCW 79 and RCW 120 star-forming regions have bright counterparts at $24.0 \mu \mathrm{m}$. The authors conclude that this large infrared excess comes from the existence of an envelope, implying that these $\mathrm{H}_{2}$ sources are in an early evolutionary phase.

However, the attribute of massive YSO relies on the additional presence of a strong IR excess. \#6, \#14 and \#11 exhibit both NIR and MIR excesses. Owing to its proximity to the bright source IRS 4 and to saturation in the IRAC maps, we can only report the NIR excess of source \#2 at subarcsecond spatial resolution. The photospheric features present in sources \#6 and \#14 seem indicative of a more evolved phase, when the central star has significantly eroded the disk and photospheric features in absorption are present. This idea is supported by their IRAC colour-colour classification: Class II in the case of \#6 and Class I for \#14 and \#11. However, the fits of the SEDs to black-body distributions indicates decreasing temperature, which could be directly related to the different spectral features.

Objects \#23, \#36, and \#18 possess featureless NIR spectra, and \#23 and \#18 show both NIR and MIR excess and a Class II IRAC classification, indicating a very young nature (Greene \& Lada 1997). Source \#36 is a deeply embedded source $\left(A_{\mathrm{V}} \sim 40 \mathrm{mag}\right)$ that displays the NIR colours of a reddened naked photosphere without any intrinsic excess. This hypothesis is supported by its not being detected in the IRAC maps. We tentatively classify \#36 as an early-type $O$ star whose shallow hydrogen features are not detected at the limited SNR of our NIR spectra.

The SED of \#26 (IRS 3) reveals a strong MIR excess that translates into the IRAC colours of a Class I object.

We have spectroscopically classified source \#12 as a Herbig Be star. The SED reveals an IR excess that rises up to $8.0 \mu \mathrm{m}$. Its NIR photometry renders a reddening-free parameter $Q=$ $(J-H)-1.70\left(H-K_{\mathrm{s}}\right)$ equivalent to -0.29 . Herbig Ae/Be star candidates cover a range $-1.38<Q<-0.22$ according to Hernández et al. (2005), reinforcing our classification. Among the identified PMS stars, only source \#58 shows significant midIR excess. However, the non detection of \#55 and \#39 in the IRAC maps at 5.8 and $8.0 \mu \mathrm{m}$ prevents further conclusions on the IR excess of these sources.

Despite being classified as OB stars, the SEDs of sources IRS 5 and \#35 exhibit strong MIR excesses (i.e. the typical colours of Class I sources). In the case of \#35, this effect could be explained by the presence of a nearby object (1'.2 away) that is not resolved in the longer wavelength IRAC channels.

\section{Discussion}

\subsection{Distance, age, and mass}

An initial estimate of the distance based on spectro-photometry by Blitz et al. (1982) situated NGC 7538 at an heliocentric distance of $2.8 \pm 0.9 \mathrm{kpc}$. Meanwhile, kinematical estimates delivered a distance for the same region as far as $3.5 \mathrm{kpc}$ (Israel et al. 1973). Russeil et al. (2007) determined the spectral type classification of two stars in the HII region, IRS $6(\mathrm{O} 9 \mathrm{~V})$, and IRS 5 $(\mathrm{O} 3 \mathrm{~V})$, inferring spectro-photometric distances of $1.6 \pm 0.17$ and $4.24 \pm 0.29 \mathrm{kpc}$, respectively. They estimated an average distance of $2.27 \pm 0.15 \mathrm{kpc}$ for the entire Sh $2-158$ region. The most recent estimate of the distance to NGC 7538 has been obtained by measuring trigonometric parallaxes of methanol masers, regularly associated with high-mass star forming regions. This study yielded a distance of $2.65_{-0.11}^{+0.12} \mathrm{kpc}$ Moscadelli et al. (2009).

Our revised optical classification of the O-type sources IRS 6 and IRS 5 described in Sect. 3.2 renders a new distance estimate to NGC 7538. Considering a classification O3V for the IRS 6 object, we derive a spectro-photometric distance of $2.99 \pm 0.5 \mathrm{kpc}$ to the cluster powering the $\mathrm{H}$ II region. Likewise, the classification of IRS 5 yields a value of $2.39 \pm 0.4 \mathrm{kpc}$. We report a final spectro-photometric distance of $2.7 \pm 0.5 \mathrm{kpc}$ to this region.

Despite the large extinction variations in the region that hampered robust age determination, Balog et al. (2004) find an older generation of stars ( 4 Myr) and a younger population of faint stars closer to $\sim 1$ Myr. A fraction of $30 \%$ of the young stars in the region around NGC 7538 was reported to exhibit a near-IR excess. An upper limit limit to the age of the cluster can be established from the most massive classified star member. According 
Table 1. Coordinates, LIRIS photometry and spectral classification of the selected objects within NGC 7538.

\begin{tabular}{|c|c|c|c|c|c|c|c|c|c|c|}
\hline Name & ID & RA (J2000) & Dec (J2000) & $J$ & $H$ & $K_{\mathrm{s}}$ & $A_{\mathrm{V}}(\mathrm{mag})$ & Class. & ST & Notes \\
\hline IRS 6 & & 231334.3 & +613014.3 & $8.71 \pm 0.02^{\dagger}$ & $8.21 \pm 0.04$ & $8.00 \pm 0.01^{\dagger}$ & $5.4 \pm 0.4$ & & $\mathrm{O} 3 \pm 1 \mathrm{~V}$ & $09 \pm 1 \mathrm{~V}^{\ddagger}$ \\
\hline IRS 5 & & 231330.3 & +613011.6 & $10.38 \pm 0.03^{\dagger}$ & $9.82 \pm 0.04^{\dagger}$ & $9.43 \pm 0.04^{\dagger}$ & $7.6 \pm 0.6$ & & $\mathrm{O} 9 \pm 1 \mathrm{~V}$ & $\mathrm{O} 3 \pm 1 \mathrm{~V}^{\ddagger}$ \\
\hline \multirow{20}{*}{ IRS 4} & \#35 & 231347.336 & +612753.74 & $18.18 \pm 0.03$ & $14.62 \pm 0.01$ & $12.55 \pm 0.01$ & $35.0 \pm 0.2$ & & B1.5-B3V & \\
\hline & \#25 & 231343.820 & +612928.37 & $13.41 \pm 0.01$ & $12.33 \pm 0.01$ & $11.83 \pm 0.02$ & $9.3 \pm 0.3$ & & B2-B7V & \\
\hline & \#17 & 231331.933 & +612747.40 & $13.83 \pm 0.01$ & $12.38 \pm 0.01$ & $11.59 \pm 0.01$ & $13.6 \pm 0.2$ & & B5-B9V & \\
\hline & \#63 & 231345.058 & +612753.88 & $18.13 \pm 0.05$ & $15.14 \pm 0.03$ & $13.18 \pm 0.01$ & - & YSO & - & $\mathrm{H}_{2}$ \\
\hline & \#6 & 231343.498 & +612937.31 & $11.97 \pm 0.01$ & $10.87 \pm 0.01$ & $9.73 \pm 0.03$ & - & YSO & - & neb \\
\hline & $\# 14$ & 231344.538 & +612715.19 & $14.89 \pm 0.02$ & $12.81 \pm 0.02$ & $11.39 \pm 0.01$ & - & YSO & - & neb, Br-abs \\
\hline & $\# 11$ & 231330.688 & +612907.47 & $17.50 \pm 0.04$ & $13.78 \pm 0.01$ & $11.15 \pm 0.01$ & - & YSO & - & $\mathrm{H}_{2}$, neb \\
\hline & \#2 & 231332.219 & +612907.32 & $11.40 \pm 0.01$ & $10.43 \pm 0.05$ & $9.43 \pm 0.09$ & - & YSO & - & neb \\
\hline & \#23 & 231334.240 & +612909.62 & $14.13 \pm 0.01$ & $12.74 \pm 0.01$ & $11.78 \pm 0.02$ & - & FL & - & \\
\hline & \#36 & 231345.380 & +612804.19 & $20.09 \pm 0.14$ & $15.25 \pm 0.03$ & $12.61 \pm 0.01$ & & FL & - & \\
\hline & $\# 18$ & 231357.268 & +612815.63 & $15.58 \pm 0.01$ & $13.15 \pm 0.01$ & $11.67 \pm 0.01$ & - & FL & - & \\
\hline & \#26 & 231343.620 & +612814.00 & $16.82 \pm 0.04$ & $13.67 \pm 0.03$ & $11.95 \pm 0.03$ & $29.7 \pm 0.7$ & H II & - & \\
\hline & $\# 12$ & 231333.537 & +612849.80 & $15.72 \pm 0.07$ & $12.92 \pm 0.07$ & $11.10 \pm 0.04$ & - & Herbig Be & - & \\
\hline & \#55 & 231338.488 & +612847.59 & $15.98 \pm 0.02$ & $13.98 \pm 0.01$ & $12.92 \pm 0.02$ & $16.0 \pm 0.3$ & PMS & G9 \pm 1 PMS & \\
\hline & \#39 & 231340.766 & +612828.20 & $14.98 \pm 0.01$ & $13.33 \pm 0.01$ & $12.54 \pm 0.01$ & $11.6 \pm 0.2$ & PMS & $\mathrm{K} 1 \pm 1 \mathrm{PMS}$ & \\
\hline & \#58 & 231331.325 & +613022.24 & $15.12 \pm 0.01$ & $13.73 \pm 0.01$ & $12.98 \pm 0.01$ & $10.3 \pm 0.2$ & PMS & $\mathrm{K} 1 \pm 1 \mathrm{PMS}$ & neb $\mathrm{Br} \gamma$ \\
\hline & \#41 & 231333.391 & +612935.77 & $14.43 \pm 0.01$ & $13.15 \pm 0.01$ & $12.56 \pm 0.01$ & $7.9 \pm 0.3$ & PMS & $\mathrm{K} 1 \pm 1 \mathrm{PMS} / \mathrm{III}$ & \\
\hline & \#15 & 231357.238 & +612747.40 & $14.95 \pm 0.01$ & $12.60 \pm 0.01$ & $11.56 \pm 0.01$ & $16.5 \pm 0.2$ & & G8 \pm 1 III & \\
\hline & \#46 & 231334.218 & +613017.64 & $14.18 \pm 0.07$ & $13.03 \pm 0.09$ & $12.66 \pm 0.08$ & $4.9 \pm 1.4$ & & $\mathrm{KO} \pm 1 \mathrm{III}$ & \\
\hline & $\# 60$ & 231344.280 & +612810.10 & $15.68 \pm 0.01$ & $13.91 \pm 0.01$ & $13.05 \pm 0.03$ & $11.9 \pm 0.6$ & & $\mathrm{~K} 2 \pm 1 \mathrm{III}$ & $\mathrm{H}_{2}$ \\
\hline IRS 7 & \#4 & 231336.801 & $\begin{array}{r}+613039.59 \\
\end{array}$ & $8.44 \pm 0.04$ & $7.63 \pm 0.04^{\dagger}$ & $7.37 \pm 0.01^{\dagger}$ & $2.6 \pm 0.6$ & & $\mathrm{~K} 0 \pm 1 \mathrm{III}$ & \\
\hline
\end{tabular}

Notes. YSO: young stellar object; FL: featureless spectrum; PMS: pre-main-sequence. ${ }^{(\dagger)}$ Photometry from the 2MASS Point Source Catalog; (‡) classification by Russeil et al. (2007) .

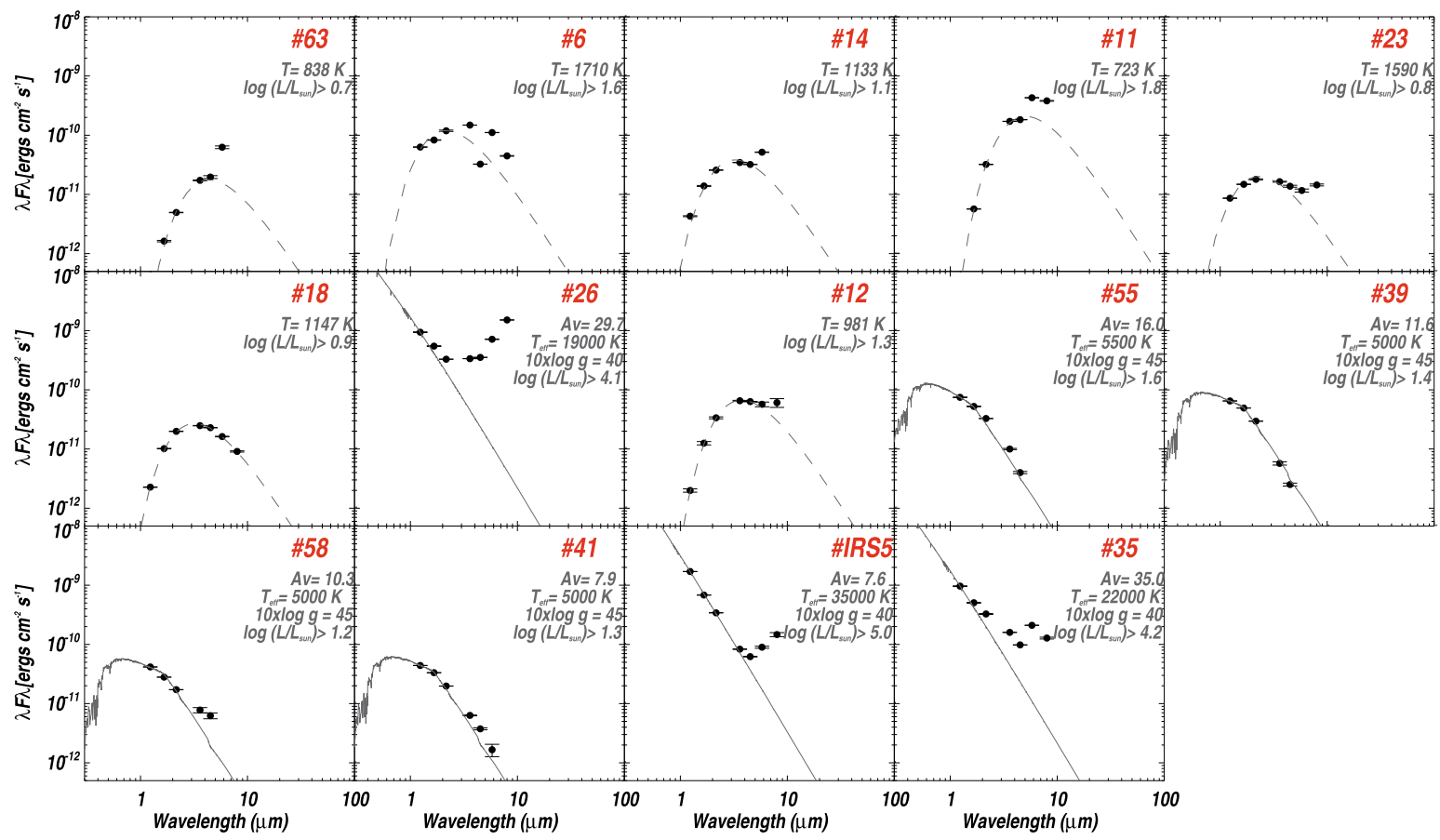

Fig. 7. SEDs of young sources in NGC 7538. Black dots with error bars are photometric measurements with LIRIS and IRAC. For the sources with a spectral type classification and an extinction estimate, the SED has been dereddened. Solid grey lines represent the Kurucz model fit of the respective spectral type classification, indicated by the $T_{\text {eff }}$ and $10 \times \log g$ labels. The area below the model in the range $0.02-1000 \mu \mathrm{m}$ corresponds to the estimated luminosity. Dashed grey lines represent the fit of a black-body distribution with the indicated temperature and luminosity.

to Schaerer \& de Koter (1997), a $60 M_{\odot}$ star (corresponding to a $\mathrm{O} 3 \mathrm{~V}$ star) has a main-sequence lifetime $\sim 2.2 \mathrm{Myr}$ before it starts the giant phase. Assuming a coeval star-formation event, the oldest stellar content in NGC 7538 must therefore be younger than $2.2 \mathrm{Myr}$.
At the other end of the mass spectrum, we can use the information derived from the PMS stars to constrain the age of the low-mass population around NGC 7538. To construct a Hertzsprung-Russell diagram (HRD), we used the spectral type to temperature conversion from Kenyon \& Hartmann (1995), 


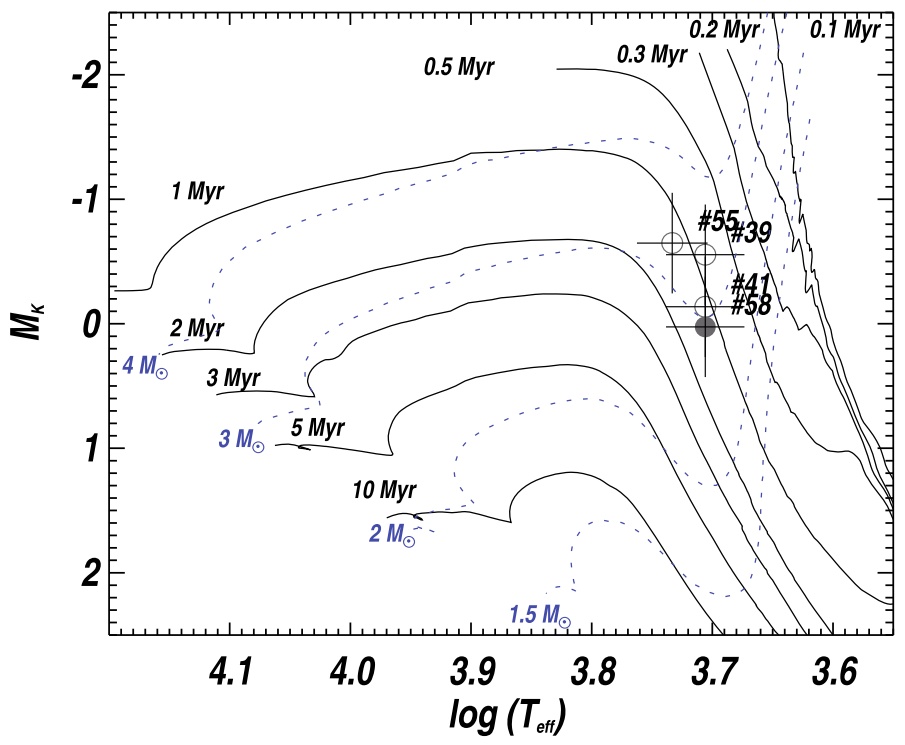

Fig. 8. Extinction corrected Hertzsprung-Russell diagram of the identified PMS stars within NGC 7538. The open symbols correspond to PMS stars without an IR excess detected in their LIRIS and IRAC photometry, while the filled ones represent those with detected IR excess. The solid lines represent the pre-main-sequence isochrones, while the dotted lines correspond to evolutionary tracks by Da Rio et al. (2009).

including the overestimates reported by Cohen \& Kuhi (1979) as temperature error. The absolute $K$ magnitudes were then calculated from the dereddened apparent brightness and assuming a distance of $2.7 \mathrm{kpc}$. The HRD of the PMS stars identified in NGC 7538 is shown in Fig. 8. We depict several pre-mainsequence evolutionary tracks in the figure and isochrones taken from Da Rio et al. (2009). The mass of these PMS stars is estimated in the range $2-4 M_{\odot}$. The comparison with the PMS isochrones yields an age range between 0.5 and $2 \mathrm{Myr}$ for the PMS identified population. We thus conclude that these two estimates result in an age range between 0.5 and 2.2 Myr for the powering cluster.

Clearly, other massive young stellar populations are also present at several locations around NGC 7538. They range from stars for which we observe photospheric spectral features and which are detectable in the optical, but also exhibit very strong IR excess (IRS 5 at region (ii)) to objects whose emission is dominated by a circumstellar disk (region (iii)), and finally to nascent stars that present ongoing outflow activity (IRS $1 / 2$ and IRS 9 in regions (iv) and (vi), respectively). These results support the previous idea of a star-forming sequence in the northwest-southeast direction that may be smoother than previously considered. The quantitative analysis of the individual ages of these populations is beyond the scope of this paper.

By assuming a Salpeter initial mass function (IMF) and extrapolating it down to $0.8 M_{\odot}$, we can calculate the stellar mass of a cluster. Nevertheless, this estimate is only meaningful for a coeval stellar population. This should be the case for the powering cluster of the HII region, with IRS 6 as the ionising star. After normalising the stellar mass distribution by the detection of one $\mathrm{O} 3 \mathrm{~V}$ star with a mass uncertainty between 47 and $64 M_{\odot}$, we obtain a total mass $\sim 1.7 \times 10^{3} M_{\odot}$ for the cluster.

\subsection{Spatial distribution}

The second most massive, identified star is IRS 5, and although it is located amidst the ionised emission, its infrared excess and proximity to the rim of the molecular cloud suggest that it may not be a member of the central cluster. Region (ii) in fact comprises a younger generation of stars. Among the spectroscopically identified B-type stars, only object \#25 is located amidst the ionised emission, while sources \#17 and \#35 are farther away. In the case of \#35, this source is located in the vicinity of the young region IRS 1-3 or (iv) and exhibits infrared excess typical of Class $0 /$ I objects.

In fact, our CMD in the left panel of Fig. 2 reveals a deficit of early B-type candidates for sources located within a radius of 30" from the O3V ionising star IRS 6 (objects marked by squares). To further study this sparseness of early B-type stars associated to the cluster, we identify B-type candidates of compatible $K_{\mathrm{s}}$-band brightness and $H-K_{\mathrm{s}} \sim 0.4 \mathrm{mag}$ in the CMD. These objects are indicated by diamonds in the left panel of Fig. 2. Analogously to our previous analysis of spectroscopically identified B-type stars, a few of the B-type candidates show a mid-IR excess (Chavarría et al., private communication) suggesting they belong to younger pockets of star formation. Other B-type candidates exhibit only photospheric emission and are located to the east and south of IRS 6, spreading beyond the boundary of the $\mathrm{H}$ II region, possibly due to a mass segregation effect.

We sketch a possible picture in which the cavity created by the ionizing stars in the molecular cloud is open in the observer's direction, and part of its stellar population is located in the foreground of the neutral molecular cloud, appearing almost aligned with other embedded younger star-forming regions. This hypothesis is supported by the work of Balog et al. (2004), who find that the stellar density peak of this region at NIR wavelengths is located at the rim of the H II region.

This projection effect is particularly important to bear in mind when isolating the stellar populations of the different pockets of star-formation that may be triggered by the expansion of $\mathrm{H}$ II regions.

\section{Summary and conclusions}

We have reported the spectro-photometric study of a sample of candidate high-mass stars in NGC 7538. We used the LIRIS instrument to obtain $J H K_{\mathrm{s}}$ photometry, and multi-object and longslit spectroscopy in the $H$ and $K$ bands at low and medium resolution. Complementary IRAC photometry of the sample has aided in the investigation of infrared excesses.

Besides the optically known O stars IRS 6 and IRS 5, whose spectral type classification we revised, we pinpointed a population of B-type stars located either at or beyond the rim of the H II region. In view of this, we confirm that IRS 6 is the main ionising source of Sh 2-158. Considering the non detection of spectral features and the lack of infrared excess, we suspect that object \#36 is a deeply embedded, early O-type star in the vicinity of IRS 1-3. However, higher signal-to-noise-ratio observations are needed to ascertain this hypothesis. We spectroscopically identified four $\mathrm{G}$ and $\mathrm{K}$ stars still contracting to the main sequence, and therefore labelled them as PMS. These stars are considered to be the precursors of A and F stars.

The observations of IRS 3, aka \#26, are consistent with its previous classification as a B-type candidate that has already started powering a compact $\mathrm{H}$ II region in a high-density environment $n_{\mathrm{e}}>10^{4} \mathrm{~cm}^{-3}$ near the protostars IRS 1-2. The outflow activity traced by the [FeII] and $\mathrm{H}_{2}$ molecular emission also support the young nature of this object.

Five of the observed sources have been classified as YSOs, with their spectra dominated either by $\mathrm{H}_{2}$ or $\mathrm{CO}$ features. Two stars display featureless NIR spectra and a prominent infrared 
excess, while object \#12 has been identified as a Herbig Be star. In general, all these YSOs are considered to be the precursors of OB stars.

We established a distinction between an older population of naked stellar photospheres and various regions of more recent star formation around NGC 7538. However, the former population spreads spatially beyond the $\mathrm{H}$ II region likely because of a projection effect, which makes it difficult to isolate the populations associated to the individual pockets of star formation. We find that most of the YSOs are concentrated either at the southern rim of the H II region or in the area around the infrared sources IRS 1-3. This, together with the known ongoing formation of the protostars in the field, reinforces the idea of a northwestsoutheast star-forming sequence. However, the study of the detailed sequence of events and causality between them cannot be addressed with the data presented.

We have estimated a distance of $2.7 \pm 0.5 \mathrm{kpc}$, an age range 0.5-2.2 Myr, and a total mass $\sim 1.7 \times 10^{3} M_{\odot}$ for the evolved cluster content within NGC 7538. The variety of NIR spectral features shown by young objects in this field makes NGC 7538 an ideal region for studying NIR spectral features of YSO for a wide range of masses and evolutionary phases in a determined environment.

Acknowledgements. We thank the referee for valuable comments that helped improve the contents of this paper. We would also like to thank Dr. Belén López for her input into the initial manuscript.

E.P is funded by the Spanish MICINN under the Consolider-Ingenio 2010 Program grant CSD2006-00070: First Science with the GTC (http://www. iac.es/consolider-ingenio-gtc). This work has been partly supported by grants AYA2007-67456-C02/AYA2008-06166-C03.

This publication makes use of data products from the Two Micron All Sky Survey, which is a joint project of the University of Massachusetts and the Infrared Processing and Analysis Center/California Institute of Technology, funded by the National Aeronautics and Space Administration and the National Science Foundation.

\section{References}

Acosta Pulido, J. A., Ballesteros, E., Barreto, M., et al. 2003, The Newsletter of the Isaac Newton Group of Telescopes, 7, 15

Balog, Z., Kenyon, S. J., Lada, E. A., et al. 2004, AJ, 128, 2942
Benjamin, R. A., Skillman, E. D., \& Smits, D. P. 1999, ApJ, 514, 307 Bik, A., Kaper, L., \& Waters, L. B. F. M. 2006, A\&A, 455, 561 Bik, A., Puga, E., Waters, L. B. F. M., et al. 2010, ApJ, 713, 833 Blitz, L., Fich, M., \& Stark, A. A. 1982, ApJS, 49, 183

Bloomer, J. D., Watson, D. M., Pipher, J. L., et al. 1998, ApJ, 506, 727 Campbell, B., \& Persson, S. E. 1988, AJ, 95, 1185

Clark, J. S., \& Steele, I. A. 2000, A\&AS, 141, 65

Cohen, M., \& Kuhi, L. V. 1979, ApJS, 41, 743

Da Rio, N., Gouliermis, D. A., \& Henning, T. 2009, ApJ, 696, 528

Davies, B., Origlia, L., Kudritzki, R., et al. 2009, ApJ, 696, 2014

Davis, C. J., Moriarty-Schieven, G., Eislöffel, J., Hoare, M. G., \& Ray, T. P. 1998, AJ, 115, 1118

Fitzpatrick, E. L. 1999, PASP, 111, 63

Geballe, T. R., Najarro, F., \& Figer, D. F. 2000, ApJ, 530, L97

Greene, T. P., \& Lada, C. J. 1996, AJ, 112, 2184

Greene, T. P., \& Lada, C. J. 1997, AJ, 114, 2157

Hamann, F., Simon, M., Carr, J. S., et al. 1994, ApJ, 436, 292

Hanson, M. M., Conti, P. S., \& Rieke, M. J. 1996, ApJS, 107, 281

Hanson, M. M., Rieke, G. H., \& Luhman, K. L. 1998, AJ, 116, 1915

Hernández, J., Calvet, N., Hartmann, L., et al. 2005, AJ, 129, 856

Israel, F. P., Habing, H. J., \& de Jong, T. 1973, A\&A, 27, 143

Kenyon, S. J., \& Hartmann, L. 1995, ApJS, 101, 117

Knödlseder, J. 2000, A\&A, 360, 539

Kraus, S., Balega, Y., Elitzur, M., et al. 2006, A\&A, 455, 521

Manchado, A., Barreto, M., Acosta-Pulido, J., et al. 2004, in SPIE Conf. Ser., 5492, 1094

Marín-Franch, A., Herrero, A., Lenorzer, A., et al. 2009, A\&A, 502, 559

Martins, F., \& Plez, B. 2006, A\&A, 457, 637

Martins, F., Pomares, M., Deharveng, L., Zavagno, A., \& Bouret, J. 2010, A\&A, 510, A32

McCaughrean, M., Rayner, J., \& Zinnecker, H. 1991, Mem. Soc. Astron. Ital., 62,715

Messineo, M., Davies, B., Ivanov, V. D., et al. 2009, ApJ, 697, 701

Meyer, M. R., Calvet, N., \& Hillenbrand, L. A. 1997, AJ, 114, 288

Moreno, M. A., \& Chavarria-K., C. 1986, A\&A, 161, 130

Moscadelli, L., Reid, M. J., Menten, K. M., et al. 2009, ApJ, 693, 406

Ojha, D. K., Tamura, M., Nakajima, Y., et al. 2004, ApJ, 616, 1042

Pestalozzi, M. R., Elitzur, M., Conway, J. E., et al. 2004, ApJ, 603, L113

Pestalozzi, M. R., Minier, V., Motte, F., et al. 2006, A\&A, 448, L57

Puga, E., Hony, S., Neiner, C., et al. 2009, A\&A, 503, 107

Rieke, G. H., \& Lebofsky, M. J. 1985, ApJ, 288, 618

Rodgers, B. M. 2001, Ph.D. Thesis, University of Washington

Russeil, D., Adami, C., \& Georgelin, Y. M. 2007, A\&A, 470, 161

Schaerer, D., \& de Koter, A. 1997, A\&A, 322, 598

Scoville, N. Z., Hall, D. N. B., Ridgway, S. T., et al. 1979, ApJ, 232, L121

Sewilo, M., Churchwell, E., Kurtz, S., Goss, W. M., \& Hofner, P. 2004, ApJ, 605,285

Tokunaga, A. T. 2000, Infrared Astronomy, ed. Cox, A. N., 143

Winston, E., Megeath, S. T., Wolk, S. J., et al. 2009, AJ, 137, 4777 